Gut, 1973, 14, 308-310

\title{
The psoas sign, hepatic angle, normal patients, and everyday practice
}

\author{
MYRON MOSKOWITZ
}

From the Department of Radiology, University of Cincinnati Medical Center, Cincinnati, Ohio, USA

SUMMARY While extensive previous references in the literature leave little doubt that disease in the posterior pararenal space can obliterate the psoas margin either in its entirety or in part, little work has been done to evaluate the frequency of when the psoas cannot be visualized in normal individuals. The data presented here indicate that as the right psoas can be expected to be absent in normal patients from 16 to $43 \%$ of the time, therefore one cannot 'lean' heavily on it as a clinical tool.

On the other hand, absence of the left psoas margin on a single film in the proper clinical setting may be sufficient to draw one's attention to the retroperitoneal space, more particularly the posterior pararenal space, as a possible site of abnormality. However, ancillary radiographic signs such as mass displacement, gas, calcium, or abnormal depositions of fat, should be carefully sought.

Preservation of either or both psoas margins certainly does not exclude by any means significant extraperitoneal disease, as can be seen even in our small anecdotal series.

The hepatic angle is absent with such frequency in normal patients that it too can only be of questionable value as a primary radiographic sign. In our normal group of patients the splenic angle was absent more frequently than it was present.

Since Hippocrates it has been traditional that physicians pass their knowledge from one generation of doctors to another, and in the past this transmission has largely consisted of empirical observations based on anecdotal experience. Over the millennia the process has gradually evolved to one based on more objective data collection. One notable pocket of resistance to this transition has been the field of diagnostic signs, ie, the laying on of hands, the gentle art of percussion and palpation, observation, olfaction, and, yes, even taste. Radiology, a relative newcomer in medicine, likewise has diagnostic signs whose roots go deep. Many of these signs have not been put to critical review. For example, one such standard, staid radiological sign, hoary with age, and honoured by use, is the loss of outline of the psoas margin. Breathes there a medical student with soul so dead who has not heard of the mystical value of this radiological sign in identifying and localizing intraabdominal disease? The literature is replete with references to the absence of a psoas margin in a wide variety of retroperitoneal abnormalities. Harper (1960), in a review of the radiographic findings in retroperitoneal tumours, includes distortion or loss of outline of one or both psoas shadows as a major feature of tumour and retroReceived for publication 8 January 1973. peritoneal inflammatory disease. Jacobson and Carter (1951), in a study of small intestinal rupture, note that obliteration of the psoas margins by extravasated gut contents 'occurs'. Wyman (1954) analysed the radiographs of 15 patients with splenic rupture and found obliterated left psoas outlines to be present twice. Toxopeus, Lucas, and Krabbenhoft (1972) in describing eight confirmed cases of retroperitoneal rupture of the duodenum note that absence of the right psoas was described twice. In a review of 130 cases of retroperitoneal fibrosis by Packham and Yates-Bell (1968) it is stated that the psoas margins as a rule are still visible on the plain film in patients with this disease. Brown, Stanbitz, Oberkircher, and Niesen (1964), on the other hand, state that radiographs may show a disappearance of fat around the kidneys and along the iliopsoas muscle. Margulis and Burhenne (1967) in their book Alimentary Tract Roentgenology point out that in appendicitis 'obliteration of the psoas margins is frequently observed'.

On the other hand, Paul and Juhl (1959) stress the diagnostic importance of seeing the psoas muscle. They state that 'the integrity of the psoas muscle borders is important in excluding retroperitoneal lesions of inflammatory or neoplastic nature', but no figures are given. 
Meyers, Whalen, Peelle, and Berne (1972), in their most recent excellent anatomical dissertation concerning the retroperitoneal space, have beautifully defined the relationships of the various extraperitoneal structures. They point out that 'loss of visualization of the psoas muscle border must be carefully evaluated, although it is of only secondary importance in the diagnosis of extraperitoneal fluid . . . localized asymmetry in properly centered films (verified on oblique projections) is the most dependable change'. Their anatomical work and one's own experience certainly would indicate that retroperitoneal disease, particularly that which is located in the posterior pararenal space, has the capacity to obliterate psoas margins. However, for any sign to have clinical, utilitarian significance one has to know how frequently and under what circumstances it may be present in normal individuals.

In the face of this formidable array of accumulated anecdotal testimony, only Elkin and Cohen (1962) have looked at this sign askance. They reviewed single and multiple abdominal films of 200 normal patients who had been prepared for excretory urography and found unequal visualization of the psoas line in $25 \%$ of patients on a single film. This figure dropped to $11 \%$ when multiple films were reviewed. In this group, $7 \frac{1}{2} \%$ of the normal patients lacked one psoas line on a single study and $2 \frac{1}{2} \%$ on multiple studies. They further came to the conclusion that the appearance of the psoas lines is generally 'not helpful in differentiating intraperitoneal from retroperitoneal masses or in the diagnosis of peritonitis'.

\section{Method}

Antero-posterior supine films of the abdomen of young (18-32-year-old) asymptomatic men were reviewed. These films were taken as part of a preemployment physical examination required by certain industries. The patients were all applying for jobs requiring heavy manual labour. Only good to excellent quality films were accepted. The x-ray examinations were done daily, but for randomization purposes only those performed on alternate Saturdays were included in this particular study. No purgatives or enemas were given.

No attempt was made to equivocate. The psoas margin was judged 'absent' if $80 \%$ or more of its length could not be seen. No further repeat film was attempted. If films were of poor technical quality they were not used.

While the study was primarily directed at checking the presence or absence of the psoas, a note was also made of the frequency with which the hepatic and splenic angles could be seen in these normal patients.

We also examined the flat films of 14 patients with retroperitoneal disease (seven pancreatitis with abscess or cyst, one ruptured left kidney, one duodenal haematoma, one retroperitoneal abscess secondary to ruptured appendix, one retroperitoneal haematoma secondary to haemophilia, two cases of retroperitoneal metastases) looking for the integrity of the psoas margin. The results are reported as anecdotal data as the sample was so small that further statistical analysis was prohibited.

\section{Discussion}

Demonstration of a psoas margin depends upon the interface which exists between the cylinder of the mass of muscle itself and the retroperitoneal fat. If there is a large amount of fat or, if the fat is spatially arranged so that it and the margin of muscle are in contact with one another and perpendicular to the beam, the margin of the muscle will be seen. Further, if there is a great deal of stool or gas in the overlying bowel, visualization of the muscle shadow will be rendered difficult. It has been said that repeat films or oblique films may be necessary to bring out a psoas margin which is at first obscure. This simple manoeuvre will apparently reduce the number of false nonvisualizing psoas margins, but, as Elkins (1962) points out, will not bring it to zero.

We evaluated the presence or absence of the psoas margin in normal patients under essentially the same conditions which all too often exist in clinical-radiological practice. Frequently the radiologist is removed both in time and space from the patients and, unfortunately, all too often from any relevant clinical history. Further, patients who are acutely ill present themselves for examination without cleansing enemas or other preparation. It is under these circumstances that films must be reviewed, decisions as to further diagnostic procedures made, and additional studies obtained, and indeed clinical diagnoses suggested.

Our data indicate that one should expect at the 0.95 confidence limit to find the right psoas margin between 59 and $85 \%$ of the time. Further the data show, at the 0.95 level, that it will be absent between 16 and $43 \%$ of the time (table I). Therefore, any diagnosis of retroperitoneal pathology based on its

\begin{tabular}{llcl}
\hline Psoas Shadow & Seen & Not Seen & Total \\
\hline Right & $37(72 \cdot 5)$ & $14(27 \cdot 5 \%)$ & $51(100 \%)$ \\
Left & $47(92 \%)$ & $4(8 \%)$ & $51(100 \%)$ \\
\hline
\end{tabular}

Table I Visualization of psoas shadows in normal patients

$\chi^{2}=6.746 ; \mathrm{n}=1 ; \mathrm{P}<0.01$

$95 \%$ confidence limits for $37 / 51$ (59.85), $95 \%$ confidence limits for $47 / 51$ (83.99), $95 \%$ confidence limits for $14 / 51(16 \cdot 43)$. 
absence on the right side will include an unacceptably high number of normals. If the diagnosis of retroperitoneal disease is excluded on the basis of seeing the right shadow, our anecdotal experience (table II) indicates that significant pathology will be frequently overlooked.

\begin{tabular}{llll}
\hline Psoas Shadow & Seen & Not Seen & Total \\
\hline Right & $11(78 \%)$ & $3(22 \%)$ & $14(100 \%)$ \\
Left & $9(64 \%)$ & $5(36 \%)$ & $14(100 \%)$ \\
\hline
\end{tabular}

Table II Visualization of psoas shadows in abnormal patients

Contrariwise, with a probability of 0.95 the psoas margin is seen more frequently on the left side in normal patients and should be expected to be seen between 83 and $99 \%$ of the time. Yet it still was absent in $8 \%$ of our normal people. In our small anecdotal series it was present in nine of 14 of those patients with retroperitoneal abnormalities.

The work of Meyers et al (1972) very clearly explains why a high percentage of 'false' negative signs occurs. If fluid is not actually located in the posterior pararenal space it is not free to dissect downward parallel and adjacent to the psoas margin.

Absence of the hepatic and splenic angle has been recommended as a sign of intraperitoneal and extraperitoneal fluid. It has been pointed out that the medial aspect of the hepatic angle 'is lost' in the presence of anterior pararenal fluid collections.

While this may be so, our data (table III) indicate that at the 0.95 confidence level the hepatic angle will not be seen in from 8 to $34 \%$ of normal healthy young males. This, of course, casts great doubt on its value as a primary radiological sign. The splenic

\begin{tabular}{llrl}
\hline Angle & Seen & Not Seen & Total \\
\hline Hepatic & $36(82 \%)$ & $8(18 \%)$ & $44(100 \%)$ \\
Splenic & $14(34 \%)$ & $27(66 \%)$ & $41(100 \%)$ \\
\hline
\end{tabular}

Table III Visualization of hepatic and splenic angles in normal patients

$95 \%$ confidence limits for $8 / 44(8 \cdot 34), 95 \%$ confidence limits for $14 / 41$ (16.43).

angle is absent so frequently in normals in our study that it is totally worthless as a sign. In my own personal experience I find the hepatic angle, like the psoas sign, of secondary help in 'shoring up' a clinico-radiological impression.

I wish to thank Mr Amin Shafie for preparing the statistical analysis of the data.

References

Brown, K. A., Staubitz, W. J., Oberkircher, O. J., and Niesen, W. C. (1964). A review of retroperitoneal fibrosis. J. Urol., 92, 323-330.

Elkin, M., and Cohen, G. (1962). Diagnostic value of the psoas shadow. Clin. Radiol., 13, 210-217.

Harper, R. A. K. (1960). Radiology in the diagnosis of retroperitoneal tumours. Clin. Radiol., 11, 69-79.

Jacobson, G., and Carter, R. A. (1951). Small intestinal rupture due to non-penetrating abdominal injury - a roentgenological study. Amer. J. Roentgenol., 66, 52-64.

Margulis, A. R., and Burhenne, H. J. (1967). Alimentary Tract Roentgenology, p. 187. Mosby, St. Louis.

Meyers, M. A., Whalen, J. P., Peelle, K., and Berne, A. S. (1972). Radiologic features of extraperitoneal effusions-an anatomic approach. Radiology, 104, 249-258.

Packham, D. A., and Yates-Bell, J. G. (1968). The symptomatology and diagnosis of retroperitoneal fibrosis: a review and presentation of case reports. Brit. J. Urol., 40, 207-222.

Paul, L. W., and Juhl, J. H. (1959). Essentials of Roentgen Interpretation. Hoeber, New York.

Toxopeus, M. D., Lucas, C. E., and Krabbenhoft, K. L. (1972). Roentgenographic diagnosis in blunt retroperitoneal duodenal rupture. Amer. J. Roentgenol., 115, 281-288.

Wyman, A. C. (1954). Traumatic rupture of the spleen. Amer. J. Roentgenol., 72, 51-63. 